\title{
Properties of the Extremal Infinite Smooth Words
}

\author{
S. Brlek ${ }^{1}$ and G. Melançon ${ }^{2}$ and G. Paquin ${ }^{1 \ddagger}$ \\ ${ }^{1}$ Laboratoire de Combinatoire et d'Informatique Mathématique \\ Université du Québec à Montréal \\ Case Postale 8888, succursale Centre-ville Montréal (QC) CANADA H3C 3P8 \\ ${ }^{2}$ Laboratoire d'Informatique, de Robotique et de Microélectronique de Montpellier \\ Université Montpellier II \\ 161 rue Ada - 34392 Montpellier Cedex 5
}

received 30 Sep 2004, revised 23 Apr 2005, accepted 10 May 2005.

\begin{abstract}
Smooth words are connected to the Kolakoski sequence. We construct the maximal and the minimal infinite smooth words, with respect to the lexicographical order. The naive algorithm generating them is improved by using a reduction of the De Bruijn graph of their factors. We also study their Lyndon factorizations. Finally, we show that the minimal smooth word over the alphabet $\{1,3\}$ belongs to the orbit of the Fibonacci word.
\end{abstract}

Keywords: Smooth words, Kolakoski sequence, Fibonacci word, Lyndon factorization

\section{Introduction}

Smooth infinite words over $\Sigma=\{1,2\}$ are connected to the Kolakoski word [Kolakoski (1965)]

$$
K=22112122122112112212112122112112122122112122121121122 \cdots,
$$

defined as the fixed point of the run-length encoding function $\Delta$. They are characterized by the property that the orbit obtained by iterating $\Delta$ is contained in $\{1,2\}^{*}$. The early work of Dekking [Dekking (1980-1981)] contains some challenging conjectures on the structure of $K$ that still remain unsolved despite the efforts devoted to the study of patterns in $K$. For instance, we know from Carpi [Carpi $(1993,1994)]$ that $K$ does contain only a finite number of squares, implying by direct inspection that $K$ is cube-free, a result that was extended in [Brlek et al. (2006)] to the infinite class $\mathcal{K}$ of smooth words. Weakley [Weakley (1989)] showed that the complexity function (number of factors of length $n$ ) of $K$ is polynomially bounded. In [Brlek and Ladouceur (2003)] a connection is established between the palindromic complexity and the recurrence of $K$. More recently, Berthé et al. [Berthé et al. (2005)] have studied smooth words

\footnotetext{
${ }^{\dagger}$ Research supported by a NSERC (Canada) grant.

$\ddagger_{\text {Corresponding author. }}$

1365-8050 @ 2007 Discrete Mathematics and Theoretical Computer Science (DMTCS), Nancy, France
} 
over arbitrary alphabets and presented some new characterizing properties about the infinite Fibonacci word. Relevant work may also be found in [Bergeron-Brlek et al. (2003)].

In this paper, we are interested by the extremal infinite smooth words, that is the minimal and the maximal ones w.r.t. the lexicographic order. The first part of the paper concerns the computation of the extremal words. We show how to improve the naive algorithm generating them, by using a reduction of the De Bruijn graph of their factors. We also study the Lyndon factorization of the extremal words. Using a result from [Brlek et al. (2006)] about the number of squares in smooth words, we show that the Lyndon factorization of the minimal infinite smooth word is a strictly decreasing sequence of Lyndon words, while the Lyndon factorization of the maximal one is strictly decreasing following the second term, since the first two Lyndon words are equal.

In the last part of this paper, we establish a link between the Fibonacci word $F$ and the minimal infinite smooth word over the alphabet $\Sigma=\{1,3\}$. More precisely,

$$
m_{\{1,3\}}=\Delta^{3}(F) .
$$

It turns out that the minimal smooth word over $\Sigma=\{1,3\}$ may be computed in linear time and we give a transducer generating it. This minimal word appeared in Berthé et al. [Berthé et al. (2005)], but the authors did not point out its minimality.

\section{Preliminaries}

Let us consider a finite alphabet of letters $\Sigma$, equipped with an order $<$ on its letters. A finite word is a finite sequence of letters

$$
w:[1 . . n] \longrightarrow \Sigma, n \in \mathbb{N}
$$

of length $n$, and $w[i]$ or $w_{i}$ denotes its $i$-th letter. The set of $n$-length words over $\Sigma$ is denoted $\Sigma^{n}$. By convention the empty word is denoted $\epsilon$ and its length is 0 . The free monoid generated by $\Sigma$ is defined by $\Sigma^{*}=\bigcup_{n>0} \Sigma^{n}$. The set of right infinite words is denoted by $\Sigma^{\omega}$ and $\Sigma^{\infty}=\Sigma^{*} \cup \Sigma^{\omega}$. Adopting a consistent notation for sequences of integers, $\mathbb{N}^{*}=\bigcup_{n \geq 0} \mathbb{N}^{n}$ is the set of finite sequences and $\mathbb{N}^{\omega}$ is those of infinite ones. Given a word $w \in \Sigma^{*}$, a factor $f$ of $w$ is a word $f \in \Sigma^{*}$ satisfying

$$
\exists x, y \in \Sigma^{*}, w=x f y .
$$

If $x=\epsilon$ (resp. $y=\epsilon)$ then $f$ is called a prefix (resp. suffix). The set of all factors of $w$, also called the language of $w$, is denoted by $F(w)$, and those of length $n$ is $F_{n}(w)=F(w) \cap \Sigma^{n}$. Finally $\operatorname{Pref}(w)$ denotes the set of all prefixes of $w$. The length of a word $w$ is $|w|$, and the number of occurrences of a factor $f \in \Sigma^{*}$ is $|w|_{f}$. A block of length $k$ is a factor of the particular form $f=\alpha^{k}$, with $\alpha \in \Sigma$. If $w=p u$, and $|w|=n,|p|=k$, then $p^{-1} w=w[k+1] . . w[n]=u$ is the word obtained by erasing $p$.

Over the restricted alphabet $\Sigma=\{1,2\}$, there is a usual length preserving morphism, the swapping of the letters, defined by $\overline{1}=2 ; \overline{2}=1$, which extends to words as follows. The complement of $u=u_{1} u_{2} \cdots u_{n} \in \Sigma^{n}$, is the word

$$
\bar{u}=\overline{u_{1}} \overline{u_{2}} \overline{u_{3}} \ldots \overline{u_{n}}
$$


Two finite words $u$ and $v$ are conjugate when there are finite words $x, y$ such that $u=x y$ and $v=y x$. The conjugacy class of a word $u$ is denoted by $[u]$, and the length is invariant under conjugacy so that it makes sense to define $|[u]|=|u|$.

We write $u \prec v$ if and only if $u$ is a proper prefix of $v$ or if there exists an integer $k$ such that $u_{i}=v_{i}$ for $i=1, \ldots, k-1$ and $u_{k}<v_{k}$. The relation $\preceq$ defined by $u \preceq v$ if and only if $u=v$ or $u \prec v$, is called the lexicographic order. That definition holds for $\Sigma^{\infty}$. Note that in general, the complementation does not preserve the lexicographic order. Indeed, when $u$ is not a proper prefix of $v$ then

$$
u \succ v \Longleftrightarrow \bar{u} \prec \bar{v} .
$$

A word $u \in \Sigma^{*}$ is a Lyndon word if for all conjugates $v$ of $u, u \prec v$. For instance, the word 11212, with conjugacy class $\{11212,12121,21211,12112,21121\}$, is a Lyndon word while 12112 is not for not being the smallest in its conjugacy class. A word of length 1 is clearly a Lyndon word. From Lothaire [Lothaire (1983)], we have the following theorem.

Theorem 1 Any non empty finite word is uniquely expressed as a non increasing product of Lyndon words.

This product is called a Lyndon factorization. Siromoney and al. [Siromoney et al. (1994)] have extended the previous theorem to infinite words. The definition of infinite Lyndon words is necessary. So they introduced infinite Lyndon words as inductive limits of sequences of finite Lyndon words. Now, let us state their result:

Theorem 2 Any infinite word $w$ is uniquely expressed as a non increasing product of Lyndon words, finite or infinite, in one of the two following forms:

either there exists an infinite non increasing sequence of finite Lyndon words $\left(l_{k}\right)_{k \geq 0}$ such that

$$
w=l_{0} l_{1} \ldots
$$

or there exist finite Lyndon words $l_{0}, \ldots, l_{m-1}(m \geq 0)$ and an infinite Lyndon word $l_{m}$ such that

$$
w=l_{0} l_{1} \ldots l_{m-1} l_{m}, \quad \text { with } l_{0} \succeq \ldots \succeq l_{m-1} \succ l_{m} .
$$

A naive algorithm is easily deduced from the definition above, but Duval [Duval (1983)] designed an algorithm which is linear with respect to the length (see also [Berstel et al. (1991)]).

The widely known run-length encoding is used in many applications as a method for compressing data. For instance, the first step in the algorithm used for compressing the data transmitted by Fax machines, consists of a run-length encoding of each line of pixels. It also was used for the enumeration of factors in the Thue-Morse sequence [Brlek (1989)]. Let $\Sigma=\{1,2\}$ be an ordered alphabet. Then every word $w \in \Sigma^{*}$ can be uniquely written as a product of factors as follows

$$
w=1^{i_{1}} 2^{i_{2}} 1^{i_{3}} \ldots
$$

where $i_{k} \geq 0$. The operator giving the size of the blocks appearing in the coding is a function $\Delta: \Sigma^{*} \longrightarrow \mathbb{N}^{*}$,

$$
\Delta(w)=i_{1} i_{2} i_{3} \cdots=\prod_{k \geq 0} i_{k},
$$


which is easily extended to infinite words as $\Delta: \Sigma^{\omega} \longrightarrow \mathbb{N}^{\omega}$. For instance, let $w=12212211$, then $w=1^{1} 2^{2} 1^{1} 2^{2} 1^{2}$, and $\Delta(w)=[1,2,1,2,2]$. Often the punctuation and the parentheses are omitted in order to manipulate the more compact notation $\Delta(w)=12122$.

This example is characteristic. Indeed, the coding integers coincide with the alphabet on which is written $w$, so that $\Delta$ can be viewed as a partial function $\Delta:\{1,2\}^{*} \longrightarrow\{1,2\}^{*}$. Although a general theory can be done over arbitrary alphabets, in the way of P. Lamas [Lamas (1995)], we restrict from hereon the study to words over the two-letter alphabet $\Sigma=\{1,2\}$ and not having 111 or 222 as factors.

The function $\Delta$ is a contraction, that is, for every word $w \in \Sigma^{*}$ we have

$$
|\Delta(w)| \leq|w|
$$

and equality holds when

$$
w \in\{\epsilon, 2\} \cdot(12)^{+} \cdot\{\epsilon, 1\} \cup\{\epsilon, 1\} \cdot(21)^{+} \cdot\{\epsilon, 2\} .
$$

Note that $\Delta$ is not bijective because $\Delta(w)=\Delta(\bar{w})$, but commutes with the mirror image $\left({ }^{\sim}\right)$, is stable under complementation $\left(^{-}\right)$and preserves palindromes.

Proposition 3 For all $u \in \Sigma^{*}$ and for all $p \in \operatorname{Pal}\left(\Sigma^{*}\right)$ the operator $\Delta$ satisfies the conditions
(a) $\Delta(\widetilde{u})=\widetilde{\Delta(u)}$;
(b) $\Delta(\bar{u})=\Delta(u)$;
(c) $\Delta(p) \in \operatorname{Pal}\left(\Sigma^{*}\right)$

The operator $\Delta$ may be iterated, provided the process is stopped when the coding alphabet changes or when the resulting word has length 1 .

Example 1 Let $w=12211211$. The successive application of $\Delta$ gives :

$$
\begin{aligned}
& \Delta^{0}(w)=12211211 \\
& \Delta^{1}(w)=12212 \\
& \Delta^{2}(w)=1211 \\
& \Delta^{3}(w)=112 \\
& \Delta^{4}(w)=21 \\
& \Delta^{5}(w)=11 \\
& \Delta^{6}(w)=2
\end{aligned}
$$

The operator $\Delta$ extends to infinite words (see [Brlek and Ladouceur (2003)]) and also to arbitrary alphabets (see [Berthé et al. (2005)]). Define the set of infinite smooth words over $\Sigma=\{1,2\}$ by

$$
\mathcal{K}=\left\{w \in \Sigma^{\omega} \mid \forall k \in \mathbb{N}, \Delta^{k}(w) \in \Sigma^{\omega}\right\},
$$

and we have the following closure properties

$$
\begin{aligned}
u \in \Delta^{k}(\Sigma) & \Longleftrightarrow \bar{u}, \widetilde{u} \in \Delta^{k}(\Sigma), \forall k \geq 0 \\
u \in \mathcal{K} & \Longleftrightarrow \bar{u} \in \mathcal{K} .
\end{aligned}
$$


The fact that $\widetilde{u}$ does not appear in statement $(7)$ is not surprising because closure by mirror image clearly requires to work with twosided infinite words.

In $\mathcal{K}$ the operator $\Delta$ has two fixpoints, namely

$$
\Delta(K)=K, \quad \Delta(1 \cdot K)=1 \cdot K
$$

where $K$ is the Kolakoski word [Kolakoski (1965)], whose first terms are

$$
K=22112122122112112212112122112112122122112122121121122 \ldots
$$

An operator closely related to $\Delta$ was introduced by M. Dekking [Dekking (1980-1981)]. It is a function $D: \Sigma^{*} \longrightarrow \Sigma^{*}$ defined as follows:

$$
D(w)= \begin{cases}\epsilon & \text { if } \Delta(w)=1 \text { or } w=\epsilon \\ \Delta(w) & \text { if } \Delta(w)=2 x 2 \text { or } \Delta(w)=2 \\ 2 x & \text { if } \Delta(w)=2 x 1 \\ x 2 & \text { if } \Delta(w)=1 x 2 \\ x & \text { if } \Delta(w)=1 x 1\end{cases}
$$

Performing $D$ amounts simply to eliminate one leading and one trailing 1, when they appear in $\Delta(w)$. This implies that $D$ is defined only for finite words. A finite word $w$ is smooth if and only if $\forall k \geq 0, D^{k}(w) \in \Sigma^{*}$.

For later use, we specialize the notion of smoothness to right-smooth words (r-smooth for short) and left-smooth words (l-smooth). To achieve this we need two extra operators defined as follows. The right derivative is a function $D_{r}: \Sigma^{*} \rightarrow \Sigma^{*}$ such that:

$$
D_{r}(w)= \begin{cases}\epsilon & \text { if } \Delta(w)=1 \text { or } w=\epsilon \\ \Delta(w) & \text { if } \Delta(w)=x 2 \\ x & \text { if } \Delta(w)=x 1\end{cases}
$$

Similarly the left derivative is a function $D_{l}: \Sigma^{*} \rightarrow \Sigma^{*}$ such that:

$$
D_{l}(w)= \begin{cases}\epsilon & \text { if } \Delta(w)=1 \text { or } w=\epsilon \\ \Delta(w) & \text { if } \Delta(w)=2 x \\ x & \text { if } \Delta(w)=1 x\end{cases}
$$

A word $w$ is $r$-smooth if $\forall k \geq 0, D_{r}^{k}(w) \in \Sigma^{*}$, and $w$ is $l$-smooth if $\forall k \geq 0, D_{l}^{k}(w) \in \Sigma^{*}$. If the word $w$ is $r$-smooth (respectively $l$-smooth), it is called a smooth prefix (respectively a smooth suffix).

Example 2 Let $w=1122121$. The successive applications of $D_{r}$ and $D_{l}$ give:

$$
\begin{aligned}
& D_{r}^{0}(w)=1122121, \quad D_{l}^{0}(w)=1122121, \\
& D_{r}^{1}(w)=2211, \quad D_{l}^{1}(w)=22111, \\
& D_{r}^{2}(w)=22, \quad D_{l}^{2}(w)=23 \text {. } \\
& D_{r}^{3}(w)=2 \text {. }
\end{aligned}
$$

The word $w$ is $r$-smooth, but not $l$-smooth because $D_{l}^{2}(w) \notin \Sigma^{*}$. 
Example 3 Let $v=12122112$. We obtain $D_{r}^{2}(v)=32$ and $D_{l}^{4}(v)=1$. So, the word $v$ is not $r$-smooth, but it is $l$-smooth.

Remark 1 There are smooth words that are neither $r$-smooth or $l$-smooth. Take for instance the word $u=1212212211212$. The successive application of $D$ gives:

$$
\begin{aligned}
& D^{1}(u)=11212211, \\
& D^{2}(u)=21122, \\
& D^{3}(u)=22 \\
& D^{4}(u)=2 .
\end{aligned}
$$

Hence the word $u$ is smooth, but it is neither $r$-smooth or $l$-smooth:

$$
\begin{array}{ll}
D_{r}^{1}(u)=111212211, & D_{l}^{1}(u)=112122111 \\
D_{r}^{2}(u)=31122 . & D_{l}^{1}(u)=21123 .
\end{array}
$$

The De Bruijn graph of order $k$ associated to a word $w \in \Sigma^{\infty}$ is a directed graph whose vertices are labeled by factors in $F_{k}(w)$, and the edges are labeled by letters $\alpha \in \Sigma$ as follows. An edge between the vertices $y$ and $z$ is labeled $\alpha$ if and only if $z=y[2 . . k] \alpha$. For example, let $F$ be the infinite Fibonacci word defined by:

$$
F=\lim _{n \rightarrow \infty} F_{n} \quad \text { where } \quad F_{0}=2, F_{1}=1, \quad \text { and } \quad \forall n \geq 2, F_{n}=F_{n-1} F_{n-2} .
$$

The first values of $F_{n}$ are:

$$
\begin{aligned}
& F_{2}=12, \\
& F_{3}=121, \\
& F_{4}=12112, \\
& F_{5}=12112121 .
\end{aligned}
$$

It is well-known that the Fibonacci word has only 4 factors of length 3: 112,121, 211,212. Thus, its De Bruijn graph of order 3 has only 4 vertices and is:

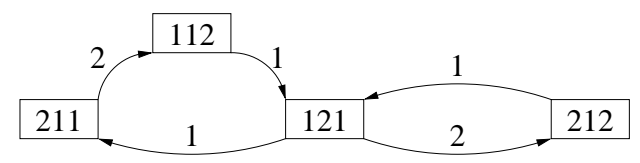

The major drawback of using these graphs is their size. Indeed the vertices of the $k$-order De Bruijn graph of the word $w$ is the set of its $k$-length factors. However it is possible to significantly reduce the size of these graphs by deleting some vertices as follows. Let $G=(V, E)$, be the $k$ order De Bruijn graph of $w \in \Sigma^{\infty}$, where $V$ is the set of the vertices and $E \subseteq V \times \Sigma \times V$, is the set of edges. The reduction of the De Bruijn graph $G$ gives a new graph $G^{\prime}=\left(V^{\prime}, E^{\prime}\right)$, where $V^{\prime} \subseteq V$ and $E^{\prime} \subseteq V^{\prime} \times \Sigma^{*} \times V^{\prime}$. The graph $G^{\prime}$ is obtained from $G$ by reducing recursively the fibers of $G$, that is to say:

$$
V^{\prime}=V-\left\{x \in V: d^{+}(x)=1 \text { and } \operatorname{succ}(x) \neq x\right\},
$$

and then, $\forall z \in \operatorname{pred}(x)$, where $x$ is a deleted vertex,

$$
E^{\prime}=E-(z, \operatorname{label}(z, x), x)-(x, \operatorname{label}(x, y), y)+(z,(\operatorname{label}(z, x) \operatorname{label}(x, y), y) .
$$


The next figure illustrates how a vertex is deleted.

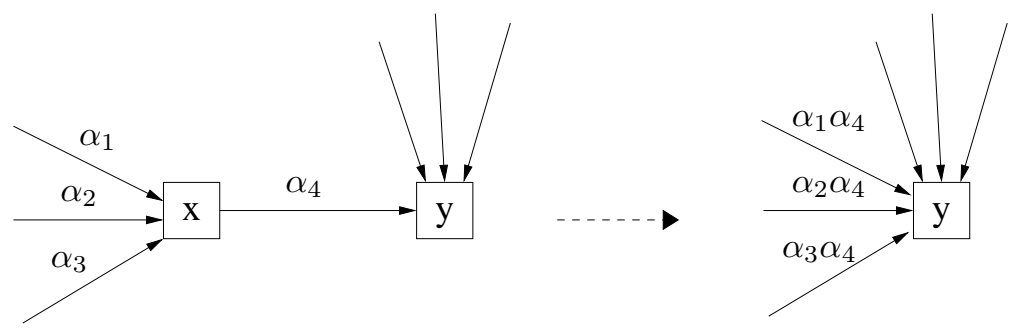

Fig. 1: Edge reduction in the De Bruijn graph

Remark 2 The order in which vertices are deleted does not influence the reduced De Bruijn graph, due to the associativity of the operation of concatenation on words. Note that in the reduced graph, the labels are words in $\Sigma^{*}$. For example, the reduced De Bruijn graph for the Fibonacci word is the following graph:

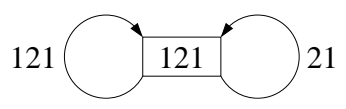

Fig. 2: Fibonacci's De Bruijn reduced graph of order 3.

\section{Results}

Let $m$ (resp. $M$ ) be the minimal (resp. maximal) smooth infinite word w.r.t the lexicographic order. From (1), it easily follows that $M=\bar{m}$, so that the computation of $m$ also yields $\bar{m}$, by simply exchanging the order on the alphabet. The simpliest algorithm that generates a prefix of length $n$ of the infinite word $m$ is the naive one and is illustrated in Figure 3.

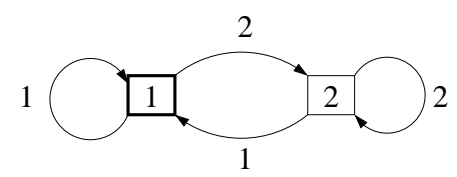

Fig. 3: Naive algorithm

For computing $m$, we need to choose at each step the edge labeled with the minimal word that keeps the word smooth. Starting at vertex 1 with the empty word, the minimal label of the edges is 1 and since 1 is smooth, we have $m[1]=1$ and we stay at vertex 1 . Next, the minimal label of the edges is still 1 and because $m[1] \cdot 1$ is smooth, we have $m[2]=1$ and stay at vertex 1 . The next step is to check the word $m[1 . .2] \cdot 1=111$ which is not smooth, because $D_{r}(111)=3$ and 
$3 \notin \Sigma$. Therefore we must choose the edge labeled 2 , and $m[1 . .3]=112$ moving to vertex 2 . In this way, an arbitrary large prefix of the word $m$ is computed. The corresponding algorithm is:

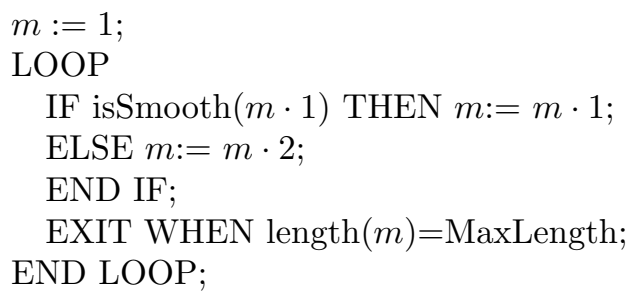

The condition isSmooth is checked with the right derivative $D_{r}$, and ensures that the prefix computed is the prefix of at least one infinite smooth word. It outputs

$$
m[1 . .24]=112112212112122112112122,
$$

for MaxLength $=24$, and consequently,

$$
\bar{m}[1 . .24]=221221121221211221221211 .
$$

With the naive algorithm, the computation of an $n$-length prefix of $m$ takes $\mathcal{O}\left(n^{2} \log (n)\right)$ : indeed, for every newly added letter to the current prefix of $m$, we have to check smoothness by applying the $D_{r}$ operator. To improve the amount of $D_{r}$ operations, it is convenient to add more than one letter at each step. For instance the reduced De Bruijn graph of order 6 for $m$ is illustrated in Figure 4.

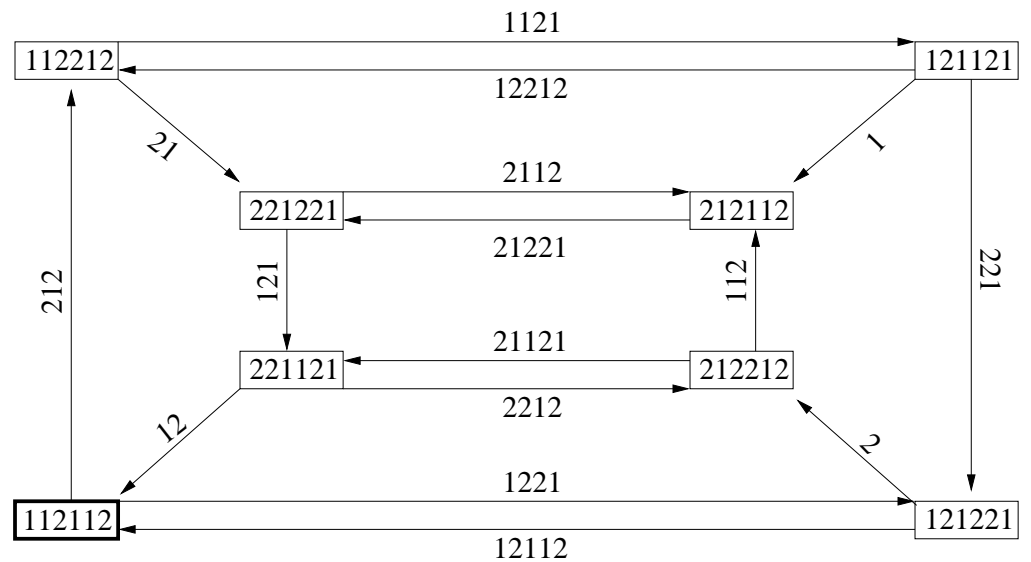

Fig. 4: Reduced 6-order De Bruijn graph of $m$ 
It translates into the following algorithm:

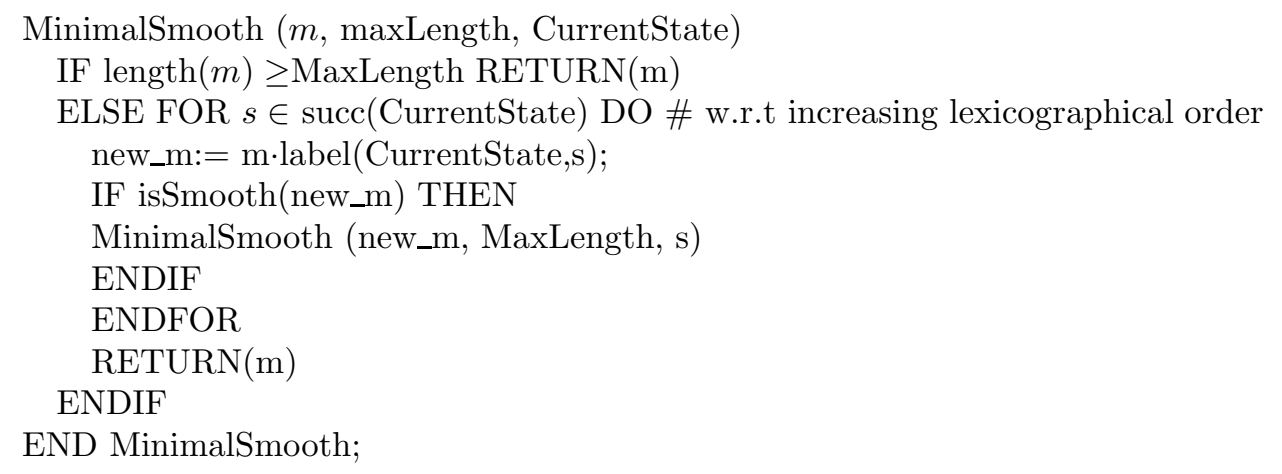

In order to produce for instance the first 100 terms of $m$, the algorithm is called by MinimalSmooth(112112, 100, InitialState). The condition isSmooth is checked with the operator of right derivative $D_{r}$. In the graph of order 6 , there are 8 vertices and the average length of the labels of the edges is 3.375 . The next table shows the growth of the number of states and the average length of the edge's labels according to the graph order.

\begin{tabular}{|c||c|c|}
\hline Order & Number of states & Average length of labels \\
\hline \hline 1 & 2 & 1 \\
2 & 2 & 1.5 \\
3 & 4 & 2.25 \\
4 & 4 & 2.25 \\
5 & 4 & 2.25 \\
6 & 8 & 3.375 \\
10 & 12 & 4.667 \\
15 & 16 & 5.062 \\
20 & 32 & 7.594 \\
25 & 36 & 8.611 \\
50 & 128 & 17.09 \\
\hline
\end{tabular}

The number of vertices as a function of the order of the graph is a polynomial function, as shown by Weakley [Weakley (1989)]. Indeed the complexity function - the number of factors of length $n$ in a smooth word - is in $\mathcal{O}\left(n^{\frac{\log 3}{\log 2}}\right)$. In the basic De Bruijn graph of the word $w$, the number of vertices is equal to the number of factors of $w$. The reduction of the De Bruijn graph reduces significantly the size of the graph without improving the complexity.

As we can see in the previous table, the average length of the labels grows logarithmically with respect to the order. Thus, augmenting the order increases the average length of the edge's labels, but also increases the number of vertices. It means that for a greater order, there are more comparisons to perform, and the growth of the average length is negligeable. So, in order to get the most efficient algorithm, we have to find the best ratio between the number of vertices 
and the average length of the labels of the edges. The algorithm was run with orders 1, 10, 20, $30,40,50,55,60$ and 65 . We found that for an order lower or equal than 50 , the increase of the order improves the algorithm. For an order bigger than 50, we found that it was less efficient. It means that for larger order, the improvement of the average length of the labels of the edges is negligeable in comparison of the increase of the number of vertices.

Let us examine the derivatives of $m$ and $\bar{m}$. Applying successively the $\Delta$ operator to $m$, we obtain:

$$
\begin{aligned}
& \Delta^{0}(m)=112112212112122112112122121122122112112122112112212112122122112112122112 \ldots \\
& \Delta^{1}(m)=212211211221211211221221211221221121121221211221221211211221221121221211 \ldots \\
& \Delta^{2}(m)=112212211212212112212212112112212112122122112112122112112212112122122112 \ldots \\
& \Delta^{3}(m)=221221121122121121221121121221211221221121121221121122121121122122121121 \cdots \\
& \Delta^{4}(m)=212212211211221211211221221211221221121221211211221221121221221121121221 \ldots \\
& \Delta^{5}(m)=112122122112122121122122112112122122112122121122122121121221121122122121 \ldots \\
& \Delta^{6}(m)=211212211211221221211212211211221211211221221211212211211212212211212212 \ldots \\
& \Delta^{7}(m)=121122122121121122122112122121121122121121221121121221221121122121121122 \ldots \\
& \Delta^{8}(m)=112212112122122112112122112112212112122122112122121122122121121122122112 \ldots \\
& \Delta^{9}(m)=221121121221211221221121121221121122121121221221121121221121122122121121 \cdots \\
& \Delta^{10}(m)=221211211221221211221221121121221211221221211211221211212211211221221211 \ldots \\
& \Delta^{11}(m)=211212212112212212112112212112122112112212212112122112112122122112112212 \ldots \\
& \Delta^{12}(m)=121121122121121221121122122121121122121121221221121122121121122122121121 \ldots \\
& \Delta^{13}(m)=112122112112212212112122112112122122112122121121122122121122122112122122 \ldots
\end{aligned}
$$

Because $m$ and $\bar{m}$ are complements, we have that $\Delta^{i}(m)=\Delta^{i}(\bar{m})$ for $i>1$. The first column word associated to $m$ is $\Phi(m)=12122121122211211121122 \cdots$, and the one associated to $\bar{m}$ is $\Phi(\bar{m})=22122121122211211121122 \cdots$. Only the first letters differ. Intuitively, because of their special constructions, the minimal and the maximal infinite smooth words should have some interesting properties. It leads to the study of their Lyndon factorization. The first factors of the Lyndon factorization of $m$ are

$\begin{aligned} f_{1}(m) & =112112212112122 \\ f_{2}(m) & =112112(\mathbf{1}) 22121122122 \\ f_{3}(m) & =1121121221(\mathbf{1}) 21122121121221221121121221121122122121121221121122121121 \cdots \\ f_{4}(m) & =112112122112112212112122122112112122112112212212112122112112(\mathbf{1}) 221221 \cdots \\ f_{5}(m) & =1121121221121122121121221221121121221121122122121121221121121221221 \cdots \\ f_{6}(m) & =1121121221121122121121(\mathbf{1}) 22122112122121121122122121122122112122121121 \cdots \\ f_{7}(m) & =11211212211211221211211221221121221211211221221211221221121(\mathbf{1}) 2122121 \cdots \\ f_{8}(m) & =112112122112112212112112212211212212112112212212112212211211212212112 \cdots \\ f_{9}(m) & =112112122112112212112112212211212212112112212(\mathbf{1}) 121221121122122121121 \cdots \\ f_{10}(m) & =1121121221121122121121122122112122121121122121121221121122122121121(\mathbf{1}) \cdots \\ f_{11}(m) & =112112122112112212112112212211212212112112212112122112112212212112112 \cdots \\ f_{12}(m) & =112112122112112212112112212211212212112112212112122112112212212112112 \cdots \\ f_{13}(m) & =112112122112112212112112212211212212112112212112122112112212212112112 \cdots\end{aligned}$

and their lengths are respectively

15, 18, 180, 910, 382, 1948, 2961, 490, 1703, 2359, 2194, 4679, 7278. 
The first factors of the Lyndon factorization of $\bar{m}$ are

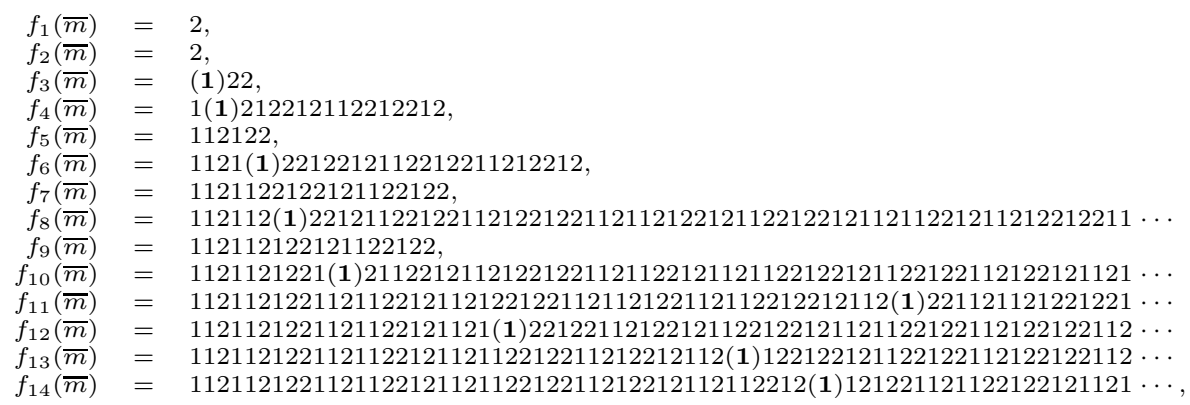

and their lengths are respectively

$$
1,1,3,17,6,27,19,80,18,180,268,1753,2107,816 \text {. }
$$

The bold letters in parenthesis are the first positions where the factor differs from the previous. It looks like if the common prefixes of two consecutive Lyndon factors are increasing.

This observation leads us to consider the existence of an infinite Lyndon word $s$ whose construction is similar to the naive construction of $m$ and $\bar{m}$. The main difference is that each time a new letter is added to the word $s$, we verify if $s$ is still a smooth suffix by applying the $D_{l}$ operator. Thus, we obtain:

$$
s=11211212211211221211211221221121221211211221211212211211221221 \cdots
$$

Now, $s$ is strictly smaller than any of its suffix: it cannot even be equal to one of its suffix, otherwise this would imply it is periodic, which is impossible because $m$ is not ultimately periodic (in fact no word in $\mathcal{K}$ is periodic, see [Brlek et al. (2006)]). This implies that $s$ is an infinite Lyndon word. The next table lists the first position $i$ in the word $s$ where it differs from $f_{k}(m)$ and $f_{k}(\bar{m})$.

\begin{tabular}{|c||c|c|}
\hline $\mathbf{k}$ & Position in $f_{k}(m)$ & Position in $f_{k}(\bar{m})$ \\
\hline \hline 1 & 7 & 2 \\
2 & 11 & 2 \\
3 & 23 & 2 \\
4 & 23 & 5 \\
5 & 23 & 5 \\
6 & 46 & 7 \\
7 & 46 & 7 \\
8 & 46 & 11 \\
9 & 68 & 11 \\
10 & 80 & 23 \\
11 & 80 & 23 \\
12 & 80 & 40 \\
13 & 142 & 46 \\
14 & 142 & 80 \\
15 & - & 248 \\
\hline
\end{tabular}


Note that in this table, "-" means that we did not calculate enough factors of $f_{k}(m)$ to complete the last line. The computation of a prefix of length 50000 was not sufficient for obtaining the 15th Lyndon factor of $m$. Observe in this table that for $k \rightarrow \infty, f_{k}(m)$ and $f_{k}(\bar{m})$ seem to converge to $s$. We could be tempted to conjecture that both $m$ and $\bar{m}$ end with $s$, which is impossible: indeed if $m=u s$ and $\bar{m}=v s$ then $m$ and $\bar{m}$ would be eventually periodic, a contradiction.

We proceed now with the study of the Lyndon factorization of $m$. We recall from [Brlek et al. (2006)] that smooth infinite words are not ultimately periodic and also cube free (see also Carpi (1994)), a consequence of the fact that the number of square factors is finite; more precisely $|\operatorname{Squares}(\mathcal{K})|=46$. It follows that $|\operatorname{Squares}(m)| \leq 46$ and, among them, the squares of Lyndon words are:

$$
1^{2},(112)^{2},(112112212)^{2},(112112212212112212211212212)^{2},(122)^{2}, 2^{2}
$$

satisfying the condition

$$
\begin{aligned}
& 1^{2} \prec(112)^{2} \prec f_{2}(m) \prec(112112212)^{2} \prec f_{1}(m) \prec \ldots \\
&(112112212212112212211212212)^{2} \prec(122)^{2} \prec 2^{2} .
\end{aligned}
$$

From the computation of the Lyndon factors $f_{1}(m)$ and $f_{2}(m)$ above, one concludes that these factors do not occur as Lyndon factors and we have the following property.

Proposition 4 Let $m=f_{1}(m) f_{2}(m) \ldots$, be the Lyndon factorization of $m$. Then

(i) $f_{1}(m)$ is the only Lyndon factor which is a smooth prefix;

(ii) $f_{i} \succ f_{i+1}$, for all $i$.

Proof: (i) By definition of a Lyndon factorization, we know that $f_{1}(m) \succeq f_{i}(m)$, for $i>1$. Earlier, we have computed the first factors of the Lyndon factorization of $m$ and have obtained that $f_{1}(m) \succ f_{2}(m)$. So, we have that for every $i \geq 2, f_{1}(m) \succ f_{i}(m)$. But, by its construction we know that $m$ is the smallest smooth prefix. Therefore, a smooth prefix smaller than $f_{1}(m)$ does not exist, and then, for all $f_{i}(m) \prec m, f_{i}(m)$ cannot be a smooth prefix and the conclusion follows.

(ii) Assume now that there exists $i$ such that $f_{i}=f_{i+1}$. Since for all $i \geq 1, f_{i}$ starts with 11 it follows that it ends with 2. From (8), it suffices to show that 1,112 do not occur as factors in the Lyndon factorization of $m$ : but in both cases it would imply that $m$ is ultimately periodic. Contradiction.

A similar statement holds in the case of the maximal smooth word $\bar{m}$.

Corollary 5 In the Lyndon factorization of $\bar{m}$,

(i) $f_{1}(\bar{m}), f_{2}(\bar{m}), f_{3}(\bar{m}), f_{5}(\bar{m}), f_{6}(\bar{m}), f_{7}(\bar{m})$ are the unique smooth prefixes;

(ii) $f_{i} \succ f_{i+1}$, for all $i \geq 2$.

Proof: (i) Whether the Lyndon factors $f_{i}(\bar{m})$, for $i \leq 7$, are smooth prefixes or not is obtained by straightforward verification, using the $D_{r}$ operator. For $i>7$, it suffices to check that $f_{8}(\bar{m}) \prec f_{1}(m)$.

(ii) Observe that $f_{8}(\bar{m}) \prec(112112212)^{2}$ in formula (8), so that the conclusion follows. 
Remark 3 In fact this property holds for any smooth infinite word in $\mathcal{K}$. Moreover, whether the Lyndon factorization of $m$ is of type (2) or (3), that is

$$
m=f_{1}(m) \cdots f_{k-1}(m) f_{k}(m)
$$

where $f_{i}(m)(i=1 \ldots k-1)$ are finite words and $f_{k}(m)$ is an infinite Lyndon word, depends on the existence of a minimal infinite suffix of $m$ (not necessarily equal to $s$ ). Would it be the case, the determination of $k$ would be a natural problem. In the same spirit, determining all infinite Lyndon suffixes becomes a relevant problem as well as the computation of a smooth word having $s$ as suffix.

Remark 4 The minimal infinite smooth word $m_{\{1,2\}}$ is not a Lyndon word. Indeed, the minimal infinite smooth word has a finite suffix smaller than every prefix of length $>6$. If every infinite smooth word contains every smooth finite factor (this is a conjectured property), then no infinite smooth word is a Lyndon word.

\section{The Fibonacci orbit and its minimal word}

Recall that the infinite Fibonacci word $F$ is defined as

$$
F=\lim _{n \rightarrow \infty} F_{n} \quad \text { where } \quad F_{0}=2, \quad F_{1}=1, \quad \text { and } \quad \forall n \geq 2, F_{n}=F_{n-1} F_{n-2} .
$$

In [Berthé et al. (2005)] it is shown that $F$ is not smooth over the alphabet $\Sigma=\{1,2\}$, but smooth over the alphabet $\Sigma=\{1,2,3\}$. The smooth tiling describing the orbit of $F$ is

$$
\begin{aligned}
\Delta^{0}(F) & =121121211211212112121121121211211212112121121121211212112112121121121 \cdots \\
\Delta^{1}(F) & =112111212111211121211121211121112121112111212111212111211121211121211 \ldots \\
\Delta^{2}(F) & =213111313111311131311131311131113131113111313111313111311131311131311 \cdots \\
\Delta^{3}(F) & =111311131311131113131113131113111313111311131311131311131113131113131 \cdots \\
\Delta^{4}(F) & =313111313111311131311131311131113131113111313111313111311131311131311 \cdots \\
\Delta^{5}(F) & =111311131311131113131113131113111313111311131311131311131113131113131 \cdots \\
\Delta^{6}(F) & =313111313111311131311131311131113131113111313111313111311131311131311 \cdots \\
\Delta^{7}(F) & =111311131311131113131113131113111313111311131311131311131113131113131 \cdots \\
\Delta^{8}(F) & =313111313111311131311131311131113131113111313111313111311131311131311 \cdots \\
\Delta^{9}(F) & =111311131311131113131113131113111313111311131311131311131113131113131 \cdots \\
\Delta^{10}(F) & =313111313111311131311131311131113131113111313111313111311131311131311 \cdots
\end{aligned}
$$

The first column word defined by $\Phi(F)[k]=\Delta^{k}(F)[0]$ is $\Phi(F)=112(13)^{\omega}$. The periodicity means that $\Delta^{k}(F)=\Delta^{k+2}(F)$ for all $k \geq 3$. It is also proved that the factors 33 and 31313 do not appear in any line word $\Delta^{k}(F)$, for $k \geq 2$.

Using a naive algorithm similar to the one described in the previous section, the first terms of the minimal infinite smooth word over $\Sigma=\{1,3\}$ are

$$
m_{\{1,3\}}=11131113131113111313111313111311131311131113131113131113 \cdots,
$$

and unexpectedly coincide with $\Delta^{3}(F)$. Then, the next theorem follows.

Theorem 6 We have $m_{\{1,3\}}=\Delta^{3}(F)$. 
To prove this theorem, a technical lemma based on the following Glueing lemma [Berthé et al. (2005)] is required.

Lemma 7 Let $u, v \in \Delta^{(*)}(k)$. If there exists an index $m$ such that, for all $i, 0 \leq i \leq m$, the last letter of $\Delta^{i}(u)$ differs from the first letter of $\Delta^{i}(v)$, and $\Delta^{i}(u) \neq 1, \Delta^{i}(v) \neq 1$, then

(i) $\Phi(u v)=\Phi(u)[0 . . m] \cdot \Phi \circ \Delta^{m+1}(u v)$;

(ii) $\Delta^{i}(u v)=\Delta^{i}(u) \Delta^{i}(v)$.

Lemma 8 The finite Fibonacci words $F_{n}$ satisfy the following conditions

(i) $\Delta^{3}\left(F_{2 n+1} \cdot 1^{-1}\right)=\Delta^{3}\left(F_{2 n} \cdot 2^{-1}\right) \cdot \Delta^{3}\left(2 \cdot F_{2 n-1} \cdot 1^{-1}\right)$;

(ii) $\Delta^{3}\left(F_{2 n} \cdot 2^{-1}\right)=\Delta^{3}\left(F_{2 n-1} \cdot 1^{-1}\right) \cdot \Delta^{3}\left(1 \cdot F_{2 n-2} \cdot 2^{-1}\right)$.

Proof: The basic recurrence $F_{n}=F_{n-1} \cdot F_{n-2}$ implies that $F_{n} \cdot a^{-1}=F_{n-1} \cdot F_{n-2} \cdot a^{-1}=$ $F_{n-1} \cdot b^{-1} \cdot b \cdot F_{n-2} \cdot a^{-1}$, where $a, b \in\{1,2\}$. It suffices now to show that the assumptions of the Glueing Lemma are satisfied in both cases.

(i) Let us check that the last letter of $\Delta^{i}\left(F_{2 n} \cdot 2^{-1}\right)$ differs from the first letter of $\Delta^{i}(2$. $\left.F_{2 n-1} \cdot 1^{-1}\right)$, for $0 \leq i \leq 3$. From the palindromic structure of $F_{n}$, we have that for $n \geq 3$, the word $F_{2 n} \cdot 2^{-1}$ has the suffix $s=121121211211$ and the word $2 \cdot F_{2 n-1} \cdot 1^{-1}$ has the prefix $p=21211212$. Applying the operators $D_{l}$ and $D_{r}$ introduced in section 2, but generalized over the alphabet $\{1,2,3\}$, to $s$ and $p$ respectively, we get:

$$
\begin{array}{ll}
D_{l}^{0}(s)=121121211211, & D_{r}{ }^{0}(p)=\mathbf{2 1 2 1 1 2 1 2 ,} \\
D_{l}{ }^{1}(s)=12111212, & D_{r}{ }^{1}(p)=\mathbf{1 1 1 2 1 1}, \\
D_{l}{ }^{2}(s)=1311 \mathbf{1}, & D_{r}{ }^{2}(p)=\mathbf{3 1}, \\
D_{l}^{3}(s)=1 \mathbf{3} . & D_{r}{ }^{3}(p)=\mathbf{1} .
\end{array}
$$

Hence, the conditions of the Glueing lemma are satisfied for all $n \geq 3$. This is easily seen also for $n=1,2$.

(ii) Again the palindromic structure implies that for all $n \geq 4$, the word $F_{2 n-1} \cdot 1^{-1}$ has the suffix $s=12112121121121211212$ and the word $1 \cdot F_{2 n-2} \cdot 2^{-1}$ has the prefix $p=1121121211211$. Applying again the $D_{l}$ and $D_{r}$ operators on $s$ and $p$ respectively, yields the result.

We proceed now with the proof of Theorem 6 .

Proof: The first step consists in proving by induction that $\Delta^{3}\left(F_{n} \cdot a^{-1}\right)$ is minimal for all $n \geq 2$. The basic cases are easily verified:

$$
F_{2} \cdot 2^{-1}=12 \cdot 2^{-1}=1 \quad \text { and } \quad \Delta(1)=\Delta^{2}(1)=\Delta^{3}(1)=1 ;
$$

a less obvious one,

$$
\begin{aligned}
F_{7} \cdot 1^{-1} & =121121211211212112121 \cdot 1^{-1}=12112121121121211212 \\
\Delta\left(F_{7} \cdot 1^{-1}\right) & =1121112121112111 \\
\Delta^{2}\left(F_{7} \cdot 1^{-1}\right) & =213111313 \\
\Delta^{3}\left(F_{7} \cdot 1^{-1}\right) & =1113111
\end{aligned}
$$


Assume now that $\Delta^{3}\left(F_{k} \cdot a^{-1}\right)$ is minimal for all $k \leq n$. By Lemma 8 ,

$$
\Delta^{3}\left(F_{n+1} \cdot a^{-1}\right)=\Delta^{3}\left(F_{n} \cdot b^{-1}\right) \cdot \Delta^{3}\left(b \cdot F_{n-1} \cdot a^{-1}\right) .
$$

There are two cases to consider: $a=1, b=2$ and $a=2, b=1$. In both cases, $\Delta^{3}\left(F_{n} \cdot b^{-1}\right)$ is minimal by induction hypothesis. It remains to establish that $\Delta^{3}\left(b \cdot F_{n-1} \cdot a^{-1}\right)$ is minimal, so that their concatenation is also minimal. If $a=1$ and $b=2$, then $\Delta^{3}\left(2 \cdot F_{n-1} \cdot 1^{-1}\right)$. In [Berthé et al. (2005)], it is shown that $\Delta^{3}(2 \cdot F)=\Delta^{3}(F)$, which implies that if $\Delta^{3}\left(F_{n-1} \cdot 1^{-1}\right)$ is minimal, then $\Delta^{3}\left(2 \cdot F_{n-1} \cdot 1^{-1}\right)$ is also minimal. The second case $(a=2$ and $b=1)$ is more involved (see [Berthé et al. (2005)] for an idea of the proof).

It suffices now to take the limit: $\Delta^{3}\left(F_{n} \cdot a^{-1}\right)$ is minimal implies that $\lim _{n \rightarrow \infty} \Delta^{3}\left(F_{n} \cdot a^{-1}\right)=$ $\Delta^{3}(F)$ is also minimal.

As in the previous section, the maximal infinite smooth word $M_{\{1,3\}}$ is the complement of $m_{\{1,3\}}$ obtained by exchanging the letters. Since $F$ and $m_{\{1,3\}}$ are in the same orbit, the next properties follow immediately from [Berthé et al. (2005)].

Corollary 9 The extremal infinite smooth words over $\Sigma=\{1,3\}$ satisfy the following conditions:

(i) $\Delta^{k}\left(m_{\{1,3\}}\right)=\Delta^{k+2}\left(m_{\{1,3\}}\right) \forall k \geq 0$;

(ii) $\Phi\left(m_{\{1,3\}}\right)=(13)^{\omega}$ and $\Phi\left(M_{\{1,3\}}\right)=3(31)^{\omega}$;

(iii) $m_{\{1,3\}}$ does not contain the factors 33 and 31313 and its complement, $M_{\{1,3\}}$, does not contain the complement factors 11 and 13131;

(iv) let $m_{\{1,3\}}=11 u$, then $\Delta\left(m_{\{1,3\}}\right)=3 u$.

From the property (iv), we get free a transducer that computes the minimal infinite smooth word $m_{\{1,3\}}$ in linear time.

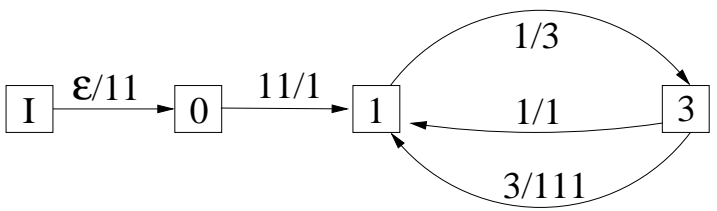

The close relation between the Fibonacci word and the minimal infinite smooth word also provides a recursive definition for $m_{\{1,3\}}$.

Proposition 10 Let $m_{\{1,3\}}=11 u$. Then the word $u$ is defined as

$$
u=\lim _{n \rightarrow \infty} u_{n} \quad \text { where } \quad u_{0}=11, \quad u_{1}=13, \quad \text { and } \quad \forall n \geq 2, u_{n}=u_{n-1} u_{n-2} .
$$

Proof: Recall that the infinite Fibonacci word $F$ can be factorized over the code $\left\{F_{i}, F_{i+1}\right\}$ for any two consecutive finite Fibonacci words. Let these two factors be $F_{3}=121$ and $F_{4}=12112$ and let $v$ be the infinite suffix defined with $F=12 v$. Then, by a simple translation of the prefix 12 in $F_{3}$ and $F_{4}$, the suffix $v$ factorizes over $C=\{112,11212\}$. By inspection of the possible cases, if $v=v_{1} v_{2} v_{3} \ldots$, where $v_{i} \in C$, then $\Delta^{3}(v)=\Delta^{3}\left(v_{1}\right) \Delta^{3}\left(v_{2}\right) \Delta^{3}\left(v_{3}\right) \cdots$. It follows that $\Delta^{3}(v)$ factorizes over the code $\{11,13\}$. Therefore, the Fibonacci recurrence also holds for the sequence $u_{n}$. 


\section{Concluding remarks}

The property that the Fibonacci word and the minimal infinite smooth word over the alphabet $\{1,3\}$ are in the same orbit is surprising and raises some natural questions.

First, since $\Phi(F))=112(13)^{\omega}$ is eventually periodic and since $\Delta^{3}(F)$ is the minimal smooth word over $\{1,3\}$, one could speculate about the properties of the words $\Phi^{-1}(P)$ where $P=u^{\omega}$ is a periodic word. For instance, if $u=a b$, where $a, b$ are odd numbers, then it turns out that $\Delta^{-k}(a)$ and $\Delta^{-k}(b)$ for $k \geq 0$ are palindromic factors. Then by using the glueing lemma with an inductive argument it follows that

$$
\Phi\left(m_{\{a, b\}}\right)=(a b)^{\omega} .
$$

Similar arguments apply for other parities. Larger finite alphabets (more than 2 letters) also deserve some attention: their study goes beyond the scope of this paper and will appear elsewhere.

\section{Acknowledgements}

The authors wish to thank the anonymous referees for the careful reading of the paper and useful comments provided. 


\section{References}

A. Bergeron-Brlek, S. Brlek, A. Lacasse, and X. Provençal. Patterns in smooth tilings. In Proc. WORDS'03, 4-th International Conference on Words Turku (Finland) September 10-13 2003, pages 370-381. TUCS General Publication 27, 2003.

J. Berstel, J. Pin, and M. Pocchiola. Mathématiques et informatique, volume 2. Combinatoire et arithmétique. McGraw-Hill, Paris, 1991.

V. Berthé, S. Brlek, and P. Choquette. Smooth words over arbitrary alphabets. Theoretical Computer Science, 341:293-310, 2005.

S. Brlek. Enumeration of factors in the thue-morse word. Discrete Applied Mathematics, 24: 83-96, 1989.

S. Brlek, S. Dulucq, A. Ladouceur, and L. Vuillon. Combinatorial properties of smooth infinite words. Theoretical Computer Science, 352:306-317, 2006.

S. Brlek and A. Ladouceur. A note on differentiable palindromes. Theoretical Computer Science, 302:167-178, 2003.

A. Carpi. Repetitions in the kolakovski sequence. Bulletin of the EATCS, 50:194-196, 1993.

A. Carpi. On repeated factors in $\mathrm{c}^{\infty}$-words. Information Processing Letters, 52:289-294, 1994.

F. Dekking. On the structure of self generating sequences. Séminaire de théorie des nombres de Bordeaux, exposé 31, 1980-1981.

J.-P. Duval. Factorizing words over an ordered aphabet. Journal of Algorithms, 4:363-381, 1983.

W. Kolakoski. Self generating runs, problem 5304. American Mathematical Monthly, 72:674, 1965.

P. Lamas. Contribution à l'étude de quelques mots infinis. Master's thesis, Université du Québec à Montréal, 1995.

M. Lothaire. Combinatorics on words. Addison Wesley, Reading MA, 1983.

G. Melançon. Viennot factorizations of infinite words. Information Processing Letters, 60:53-57, 1996.

G. Melançon. Lyndon factorization of sturmian words. Discrete Mathematics, 210:137-149, 2000.

R. Siromoney, L. Matthew, V. R. Dare, and K. G. Subramanian. Infinite lyndon words. Information Processing Letters, 50:101-104, 1994.

W. D. Weakley. On the number of $c^{\infty}$-words of each length. Journal Combinatorial Theory Series A, 51(1):55-62, 1989. 
